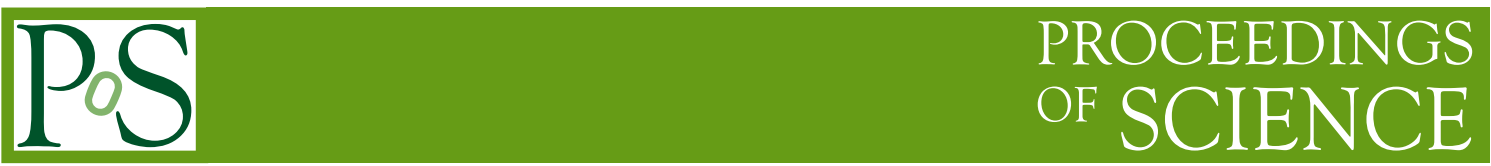

\title{
Cutoff effects on lattice nuclear forces
}

\section{Takumi Doi*}

Theoretical Research Division, Nishina Center, RIKEN, Wako 351-0198, Japan

E-mail: doidribf.riken.jp

for HAL QCD Collaboration

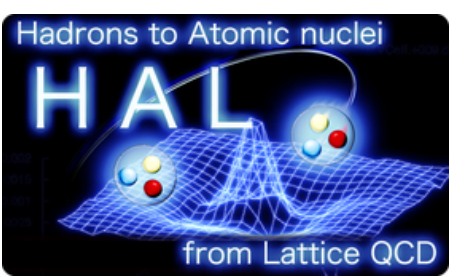

We present a lattice QCD study for the cutoff effects on nuclear forces. Two-nucleon forces are determined from Nambu-Bethe-Salpeter (NBS) wave functions using the HAL QCD method. Lattice QCD simulations are performed employing $N_{f}=2$ clover fermion configurations at three lattice spacings of $a=0.108,0.156,0.215 \mathrm{fm}$ on a fixed physical volume of $L^{3} \times T \simeq(2.5 \mathrm{fm})^{3} \times 5 \mathrm{fm}$ with a large quark mass corresponding to $m_{\pi} \simeq 1.1 \mathrm{GeV}$. We observe that while the discretization artifact appears at the short range part of potentials, it is suppressed at the long distance region. The cutoff dependence of the phase shifts and scattering length is also presented.

31st International Symposium on Lattice Field Theory - LATTICE 2013

July 29 - August 3, 2013

Mainz, Germany

\footnotetext{
* Speaker.
} 


\section{Introduction}

Nuclear forces, the interactions among nucleons, serve as the cornerstone in nuclear physics. While they have been traditionally determined through the scattering experiments, theoretical understanding of them from the fundamental theory, Quantum Chromodynamics (QCD), has not been established yet. In the last several years, substantial effort has been devoted to determine nuclear interactions using lattice QCD simulations. A conventional approach is to calculate an energy spectrum of a two-nucleon $(2 \mathrm{~N})$ system on the lattice and convert it to the scattering phase shift at the corresponding energy through the Lüscher's finite volume formula [四]. Latest lattice QCD results in this approach are given in, e.g., Refs. [2], [3].

Recently, a novel approach was proposed to determine nuclear forces on the lattice [ [G, []]. In this approach, now called as the HAL QCD method, nuclear forces (or potentials) are directly obtained from Nambu-Bethe-Salpeter (NBS) wave functions calculated in lattice simulations. In particular, the extension to the "time-dependent" HAL QCD method has an advantageous feature that energy-independent (non-local) potentials can be extracted without replying on the ground state saturation [回. Once potentials are obtained, scattering parameters such as phase shifts and scattering length can be calculated by solving the Schrödinger equation in infinite volume at arbitrary energies below the inelastic threshold. Resultant (parity-even) nuclear potentials are found to have desirable features such as attractive wells at long and medium distances and central repulsive cores at short distance. The method has been successfully applied to more general hadron interactions,

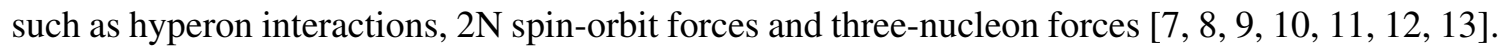
See Ref. [14]] for a recent review.

Toward the quantitative determination of nuclear forces, systematic uncertainties in lattice simulations should be carefully examined, such as the effect of discretization artifact. There have been, however, no work which performs the continuum extrapolation on nuclear interactions in Lüscher's method nor in the HAL QCD method. The aim of this work is to perform the first systematic study for the lattice cutoff dependence of nuclear interactions. In particular, in the HAL QCD method, it could be easier to understand how the discretization artifact, which has intrinsically short-range nature, affects the lattice QCD results since the spacial information remains in this method. It is also interesting to examine how the characteristic feature in lattice nuclear potentials such as repulsive cores at short distance are stable against changing the lattice cutoff. Therefore, in this study, we calculate nuclear potentials in the HAL QCD method at three different lattice spacings, with the other lattice parameters such as physical volume size and quark masses fixed. Solving the Schrödinger equation with obtained potentials, the cutoff dependence of scattering phase shifts and scattering length is also studied.

\section{Formalism}

We briefly explain the framework of the HAL QCD method [15, 6, ㄴ]]. We consider the (equaltime) NBS wave function in the center-of-mass frame,

$$
\phi_{W}(\vec{r}) \equiv\langle 0|N(\vec{r}, 0) N(\overrightarrow{0}, 0)| 2 N, W\rangle_{\text {in }},
$$


where $N$ is the nucleon operator and $|2 N, W\rangle_{\text {in }}$ denotes the asymptotic in-state of the $2 \mathrm{~N}$ system at the total energy of $W=2 \sqrt{k^{2}+m_{N}^{2}}$ with the nucleon mass $m_{N}$ and the relative momentum $k \equiv|\vec{k}|$, and we consider the elastic region, $W<W_{\text {th }}=2 m_{N}+m_{\pi}$. For simplicity, we omit other quantum numbers such as spinor/flavor indices. The most important property of the NBS wave function is that it has a desirable asymptotic behavior [U, [1, [15, [16, [17],

$$
\phi_{W}(\vec{r}) \propto \frac{\sin \left(k r-l \pi / 2+\delta_{l}^{W}\right)}{k r}, \quad r \equiv|\vec{r}| \rightarrow \infty,
$$

where $\delta_{l}^{W}$ is the scattering phase shift with the orbital angular momentum $l$. Exploiting this feature, we define the (non-local) $2 \mathrm{~N}$ potential, $U\left(\vec{r}, \vec{r}^{\prime}\right)$, through the following Schrödinger equation,

$$
H_{0} \phi_{W}(\vec{r})+\int d \vec{r}^{\prime} U\left(\vec{r}, \vec{r}^{\prime}\right) \phi_{W}\left(\vec{r}^{\prime}\right)=E_{W} \phi_{W}(\vec{r})
$$

where $H_{0}=-\nabla^{2} /(2 \mu)$ and $E_{W}=k^{2} /(2 \mu)$ with the reduced mass $\mu=m_{N} / 2$. It is evident that $U\left(\vec{r}, \vec{r}^{\prime}\right)$ defined in this way is faithful to the phase shift by construction. In addition, it has been proven that one can construct $U\left(\vec{r}, \vec{r}^{\prime}\right)$ in an energy-independent way [[5, [4]]. These points guarantee that once potentials are obtained, one can determine the phase shifts at arbitrary energies below the inelastic threshold. Furthermore, it is found that the energy-independence of potentials can

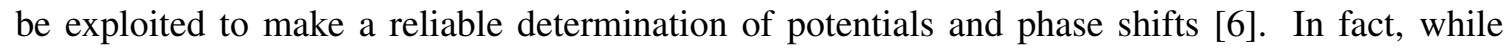
the original equation (2.3) requires the determination of $\phi_{W}$ for each energy $W$, by e.g., ground state saturation, one can show that the same $U\left(\vec{r}, \vec{r}^{\prime}\right)$ can be extracted from the "time-dependent" Schrödinger equation

$$
H_{0} \psi(\vec{r}, t)+\int d \vec{r}^{\prime} U\left(\vec{r}, \vec{r}^{\prime}\right) \psi\left(\vec{r}^{\prime}, t\right)=\left(-\frac{\partial}{\partial t}+\frac{1}{4 m_{N}} \frac{\partial^{2}}{\partial t^{2}}\right) \psi(\vec{r}, t)
$$

even without the ground state saturation, where $\psi(\vec{r}, t) \equiv G(\vec{r}, t) / e^{-2 m_{N} t}$ and $G(\vec{r}, t)$ is a four-point correlation function, $G(\vec{r}, t) \equiv \frac{1}{L^{3}} \sum_{\vec{R}}\langle 0|(N(\vec{R}+\vec{r}) N(\vec{R}))(t) \overline{(N N)}(t=0)| 0\rangle$. This "time-dependent" HAL QCD method is particularly useful for nuclear systems, since the ground state saturation becomes more and more difficult at lighter quark masses and larger lattice volumes [ [6].

In practical lattice calculations, it is difficult to handle the non-locality of the potential directly, so we employ the derivative expansion of the potential, $U\left(\vec{r}, \vec{r}^{\prime}\right)=\left[V_{C}(r)+V_{T}(r) S_{12}+V_{L S}(r) \vec{L} \cdot \vec{S}+\right.$ $\left.\mathscr{O}\left(\nabla^{2}\right)\right] \delta\left(\vec{r}-\vec{r}^{\prime}\right)$, where $V_{C}, V_{T}$ and $V_{L S}$ are the central, tensor and spin-orbit potentials, respectively, with the tensor operator $S_{12}$. In Ref. [四], the convergence of the derivative expansion is examined in parity-even channel, and it is shown that the leading terms, $V_{C}$ and $V_{T}$, dominate the potential at low energies.

The HAL QCD method can be extended to systems above the inelastic threshold [ए8, [प] as well as multi-particle systems [20]

\section{Lattice QCD setup and Numerical results}

We employ $N_{f}=2$ dynamical configurations with mean field improved clover fermion and RG-improved gauge action generated by CP-PACS Collaboration [ㅁ] ]. The measurements are 


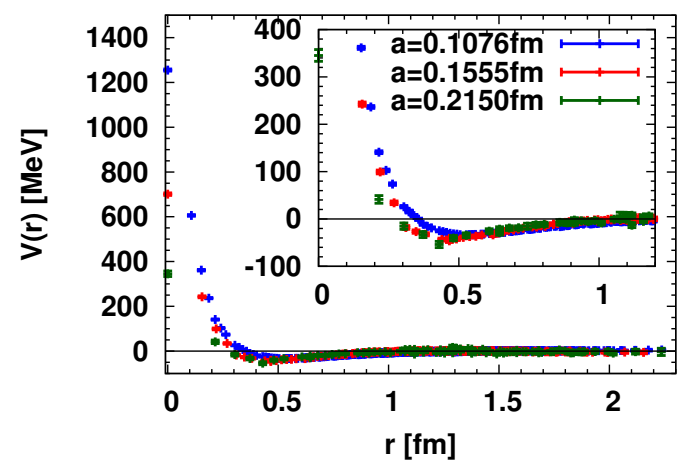

Figure 1: Nuclear central potential $V_{C}(r)$ in ${ }^{1} S_{0}$ channel obtained at three different lattice cutoffs.

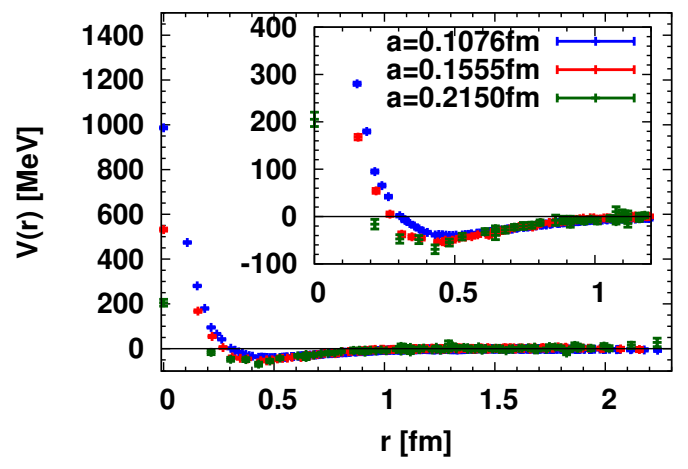

Figure 2: Nuclear central potential $V_{C}(r)$ in ${ }^{3} S_{1}-$ ${ }^{3} D_{1}$ channel.

performed at three different bare couplings $\beta=1.80,1.95,2.10$, which corresponds to the lattice spacings $a=0.2150,0.1555,0.1076 \mathrm{fm}$, respectively. The physical lattice size is $L^{3} \times T \simeq$ $(2.5 \mathrm{fm})^{3} \times 5 \mathrm{fm}$, and the hadron masses are $m_{\pi} \simeq 1.1 \mathrm{GeV}$ and $m_{N} \simeq 2.2 \mathrm{GeV}$. We use the wall quark source with Coulomb gauge fixing. In order to enhance the statistics, we repeat the measurement using different source time slices and the forward and backward propagations are averaged. Computational cost in the Wick and color/spinor contractions are reduced by the unified contraction algorithm [22]. The simulation parameters are tabulated in Tab. $\mathbb{W}$. The cutoff dependence for $I=2 \pi \pi$ interaction with the same configurations has been investigated in Ref. [23]].

In Fig. W, we plot the nuclear central potential in ${ }^{1} S_{0}$ channel for each lattice cutoff. In the evaluation of Eq. (2.4), we omit the second derivative term in $t$, which corresponds to the relativistic correction and is expected to have marginal effect at heavy quark mass [目]. Shown in Figs. $\square$ and [3] are the central and tensor potentials in ${ }^{3} S_{1}-{ }^{3} D_{1}$ channel, respectively.

Comparing the results at three different cutoffs, we observe non-negligible cutoff dependence at the short distance part of the potentials, while the cutoff dependence at long distance region is suppressed. This is a natural consequence that the discretization effect has intrinsically short-range nature. We note that a similar observation is obtained in the potential of $I=2 \pi \pi$ system, where the violation of rotation symmetry due to finite lattice spacing is found at short distance [24].

It is also interesting that repulsive cores in central forces are enhanced on a finer lattice. This tendency is consistent with the analyses by the operator product expansion (OPE), where the repulsive cores are predicted to diverge in the limit of $r \rightarrow 0$ [25]. We, however, note that even the finest lattice $(a=0.1076 \mathrm{fm})$ may not be sufficiently fine to quantitatively examine these perturbative

\begin{tabular}{ccccccccc}
\hline \hline$\beta$ & $L^{3} \times T$ & $a[\mathrm{fm}]$ & $L a[\mathrm{fm}]$ & $\kappa_{u d}$ & $m_{\pi} a$ & $m_{N} a$ & $N_{\text {conf }}$ & $N_{\text {src }}$ \\
\hline 1.80 & $12^{3} \times 24$ & $0.2150(22)$ & $2.580(26)$ & 0.14090 & $1.1562(4)$ & $2.262(2)$ & 640 & 24 \\
1.95 & $16^{3} \times 32$ & $0.1555(17)$ & $2.489(27)$ & 0.13750 & $0.8934(4)$ & $1.695(1)$ & 598 & 32 \\
2.10 & $24^{3} \times 48$ & $0.1076(13)$ & $2.583(31)$ & 0.13570 & $0.6301(3)$ & $1.182(1)$ & 798 & 48 \\
\hline \hline
\end{tabular}

Table 1: Lattice simulation parameters. $N_{\text {conf }}$ is the number of configurations, and $N_{\text {src }}$ is the number of sources per each configuration in the measurement. 


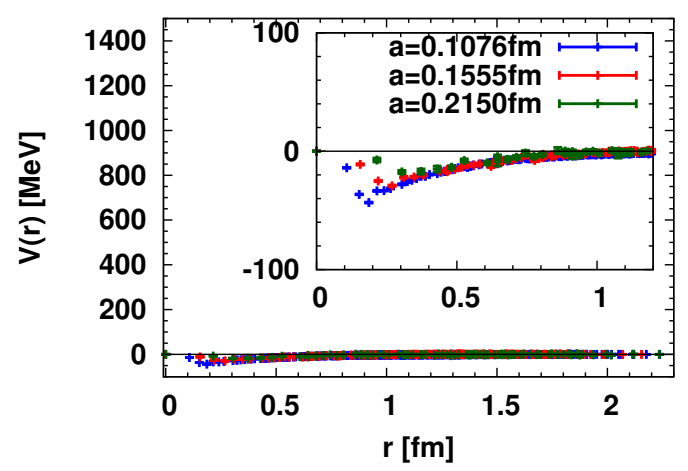

Figure 3: Nuclear tensor potential $V_{T}(r)$ in ${ }^{3} S_{1}-$ ${ }^{3} D_{1}$ channel.

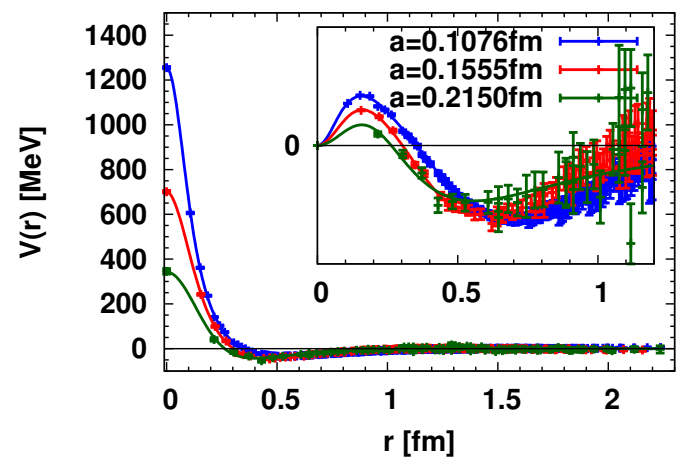

Figure 4: The fit of $V_{C}(r)$ in ${ }^{1} S_{0}$ channel. The inner figure shows $r^{2} V_{C}(r)$ to include phase space factor.

behaviors. In the tensor force, we observe kink structures at $r \sim 0.2-0.3 \mathrm{fm}$ (depending on $a$ ), and the results from different cutoffs agree if the distance $r$ is longer than the kink position, while the deviation is observed at shorter distance than the kink. In fact, we have been observing similar kink structures in tensor forces in various baryon-baryon interactions with a variety of lattice setup [14]]. The systematic study in this report indicates that these kink structures are associated with the discretization artifact.

While the lattice cutoff dependence in potentials looks sizable at short distance, it is important to realize that such effect is expected to be suppressed in physical observables such as phase shifts and scattering length, because of the phase space factor of $\propto r^{2}$. In order to make a quantitative study, we calculate the phase shifts and scattering length in ${ }^{1} S_{0}$ channel. In Fig. 团, we show the potential together with their fitted values where we employ the fitting function used in [W]. Shown in the inner figure is the potential multiplied by $r^{2}$ to account for the phase space factor. One can observe that the discretization artifact is effectively suppressed as discussed above.

By solving the Schrödinger equation using the fitted potential in infinite volume, we obtain the phase shifts. In Fig. [1, we plot the phase shifts in terms of the laboratory energy where the bands correspond to the statistical fluctuations. The qualitative behaviors are found to be similar to the experimental phase shifts. We observe that the results from different cutoffs agree at low energies within the statistical errors. On the other hand, there exists a deviation at high energies, where phase shifts become smaller on a finer lattice. These behaviors can be understood by recalling that the cutoff dependence appears only at short range part of the potential, and the repulsive core is enhanced on a finer lattice. In Fig. G, we show preliminary results for the scattering length $a\left({ }^{1} S_{0}\right)=\lim _{k \rightarrow 0} \tan \delta(k) / k$ against the lattice spacing $a$, where the error is statistical only. Since the scattering length represents the low-energy phenomena, the cutoff dependence is found to be negligible compared to the statistical errors. Detailed studies for the systematic uncertainties for phase shifts and scattering length are in progress.

We thank authors and maintainers of CPS++[26], whose modified version is used in this study. We also thank CP-PACS Collaboration and ILDG/JLDG [L7] for providing gauge configurations. The numerical simulations have been performed on SR16000 and Blue Gene/L at KEK, T2K at University of Tsukuba, SR16000 at YITP in Kyoto University and FX10 at University of Tokyo. 


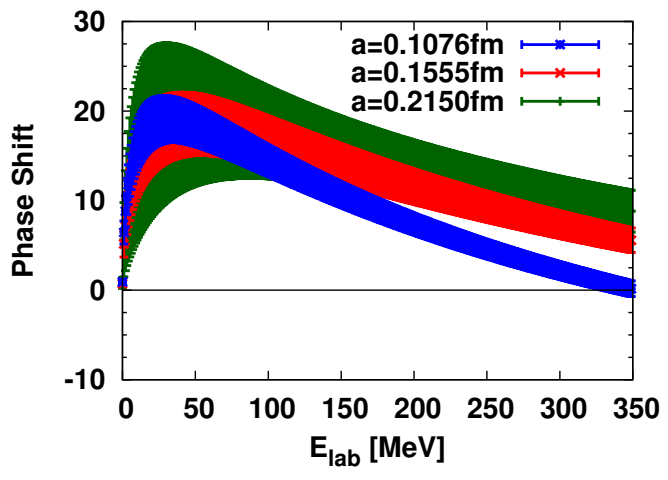

Figure 5: Phase shifts in ${ }^{1} S_{0}$ channel in terms of the laboratory energy.

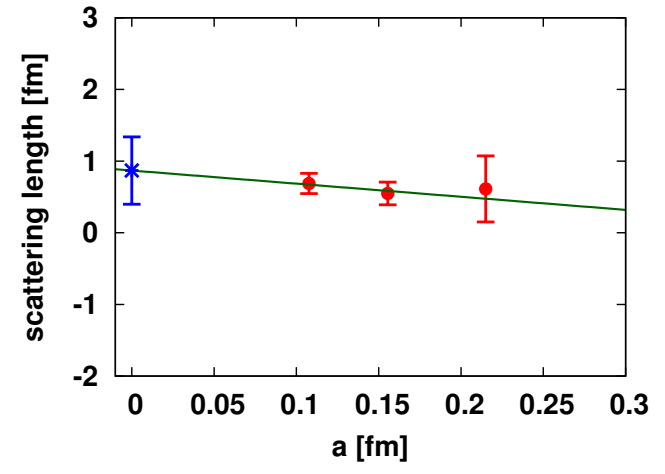

Figure 6: The scattering length in ${ }^{1} S_{0}$ channel against the lattice spacing $a$. The blue point corresponds to the result in the continuum limit obtained by the linear extrapolation against $a$.

This research is supported in part by MEXT Grant-in-Aid for Scientific Research (No. 24740146), for Scientific Research on Innovative Areas (No. 2004: 20105001, 20105003) and SPIRE (Strategic Program for Innovative REsearch).

\section{References}

[1] M. Lüscher, Commun. Math. Phys. 105 (1986) 153, ibid., Nucl. Phys. B 354 (1991) 531.

[2] T. Yamazaki, K. -I. Ishikawa, Y. Kuramashi and A. Ukawa, Phys. Rev. D 86 (2012) 074514 [arXiv:1207.4277 [hep-lat]] and refereces therein.

[3] S. R. Beane, E. Chang, S. D. Cohen, W. Detmold, P. Junnarkar, H. W. Lin, T. C. Luu and K. Orginos et al., Phys. Rev. C 88 (2013) 024003 [arXiv:1301.5790 [hep-lat]] and refereces therein.

[4] N. Ishii, S. Aoki and T. Hatsuda, Phys. Rev. Lett. 99 (2007) 022001 [nucl-th/0611096].

[5] S. Aoki, T. Hatsuda and N. Ishii, Prog. Theor. Phys. 123 (2010) 89 [arXiv:0909.5585 [hep-lat]].

[6] N. Ishii et al. [HAL QCD Collaboration], Phys. Lett. B 712 (2012) 437 [arXiv:1203.3642 [hep-lat]].

[7] H. Nemura, N. Ishii, S. Aoki and T. Hatsuda, Phys. Lett. B673 (2009) 136 [arXiv:0806.1094 [nucl-th]].

[8] T. Inoue et al. [HAL QCD Collaboration], Prog. Theor. Phys. 124 (2010) 591 [arXiv:1007.3559 [hep-lat]].

[9] T. Inoue et al. [HAL QCD Collaboration], "Bound H-dibaryon in Flavor SU(3) Limit of Lattice QCD,” Phys. Rev. Lett. 106 (2011) 162002 [arXiv:1012.5928 [hep-lat]].

[10] K. Murano, N. Ishii, S. Aoki and T. Hatsuda, Prog. Theor. Phys. 125 (2011) 1225 [arXiv:1103.0619 [hep-lat]].

[11] T. Inoue et al. [HAL QCD Collaboration], Nucl. Phys. A 881 (2012) 28 [arXiv:1112.5926 [hep-lat]].

[12] K. Murano, N. Ishii, S. Aoki, T. Doi, T. Hatsuda, Y. Ikeda, T. Inoue and H. Nemura et al., arXiv:1305.2293 [hep-lat]. 
[13] T. Doi et al. [HAL QCD Coll.], Prog. Theor. Phys. 127 (2012) 723 [arXiv:1106.2276 [hep-lat]].

[14] S. Aoki et al. [HAL QCD Collaboration], Prog. Theor. Exp. Phys. 2012 (2012) 01A105 [arXiv:1206.5088 [hep-lat]].

[15] C. J. D. Lin et al., Nucl. Phys. B 619 (2001) 467 [arXiv:hep-lat/0104006].

[16] S. Aoki et al. [CP-PACS Collaboration], Phys. Rev. D 71 (2005) 094504 [arXiv:hep-lat/0503025].

[17] N. Ishizuka, PoS LAT2009 (2009) 119 [arXiv:0910.2772 [hep-lat]].

[18] S. Aoki et al. [HAL QCD Coll.], Proc. Jpn. Acad. Ser. B 87 (2011) 509 [arXiv:1106.2281 [hep-lat]].

[19] S. Aoki, B. Charron, T. Doi, T. Hatsuda, T. Inoue and N. Ishii, Phys. Rev. D 87 (2013) 034512 [arXiv:1212.4896 [hep-lat]].

[20] S. Aoki, N. Ishii, T. Doi, Y. Ikeda and T. Inoue, Phys. Rev. D 88 (2013) 014036 [arXiv:1303.2210 [hep-lat]].

[21] A. Ali Khan et al. [CP-PACS Collab.], Phys. Rev. D 65 (2002) 054505 [E: D 67 (2003) 059901].

[22] T. Doi and M. G. Endres, Comput. Phys. Commun. 184 (2013) 117 [arXiv:1205.0585 [hep-lat]].

[23] T. Yamazaki et al. [CP-PACS Collaboration], Phys. Rev. D 70 (2004) 074513 [hep-lat/0402025].

[24] T. Kurth, N. Ishii, T. Doi, S. Aoki and T. Hatsuda, arXiv:1305.4462 [hep-lat].

[25] S. Aoki, J. Balog and P. Weisz, JHEP 1005 (2010) 008 [arXiv:1002.0977 [hep-lat]].

[26] Columbia Physics System (CPS), "http://qcdoc.phys .columbia.edu/cps .html"

[27] "http://www.lqcd.org/ildg", "http://www.jldg.org" 